Print ISSN: 2288-4637 / Online ISSN 2288-4645

doi:10.13106/jafeb.2020.vol7.no10.009

\title{
Hybrid Model Approach to the Complexity of Stock Trading Decisions in Turkey
}

\author{
Seyma CALISKAN CAVDAR ${ }^{1}$, Alev Dilek AYDIN ${ }^{2}$
}

Received: July 25, 2020 Revised: August 23, 2020 Accepted: August 29, 2020

\begin{abstract}
The aim of this paper is to predict the Borsa Istanbul (BIST) 30 index movements to determine the most accurate buy and sell decisions using the methods of Artificial Neural Networks (ANN) and Genetic Algorithm (GA). We combined these two methods to obtain a hybrid intelligence method, which we apply. In the financial markets, over 100 technical indicators can be used. However, several of them are preferred by analysts. In this study, we employed nine of these technical indicators. They are moving average convergence divergence (MACD), relative strength index (RSI), commodity channel index (CCI), momentum, directional movement index (DMI), stochastic oscillator, on-balance volume (OBV), average directional movement index (ADX), and simple moving averages (3-day moving average, 5-day moving average, 10-day moving average, 14-day moving average, 20-day moving average, 22-day moving average, 50-day moving average, 100-day moving average, 200-day moving average). In this regard, we combined these two techniques and obtained a hybrid intelligence method. By applying this hybrid model to each of these indicators, we forecast the movements of the Borsa Istanbul (BIST) 30 index. The experimental result indicates that our best proposed hybrid model has a successful forecast rate of $75 \%$, which is higher than the single ANN or GA forecasting models.
\end{abstract}

Keywords: Artificial Neural Network, Genetic Algorithm, Technical İndicators, Hybrid Models, Borsa Istanbul (BIST)

JEL Classification Code: G10, G15, G17

\section{Introduction}

Forecast of stock market index is very important for the development of effective trading strategies. The selection of attractive stocks and the determination of the most appropriate buy and sell decisions are of utmost importance to ensure a good return for the domestic as well as the international investors. Therefore, an effective trading strategy will give the trader competitive advantage over others as he or she can identify the best performing stocks with the most

${ }^{1}$ First Author. Associate Professor, Department of Economics. Faculty of Economics and Administrative Sciences, Dogus University, Istanbul, Turkey. E-mail: scaliskan@dogus.edu.tr

${ }^{2}$ Corresponding Author. Associate Professor, Department of International Trade and Business, Faculty of Business, Halic University, Istanbul, Turkey [Postal Adress: Sutluce Mah. Imrahor Cad. No. 82, 34050, Beyoglu, Istanbul, Turkey] E-mail: dilekaydin@halic.edu.tr

(c) Copyright: The Author(s)

This is an Open Access article distributed under the terms of the Creative Commons Attribution Non-Commercial License (https://creativecommons.org/licenses/by-nc/4.0/) which permits unrestricted non-commercial use, distribution, and reproduction in any medium, provided the original work is properly cited. accurate decisions concerning when to buy or sell. Hence, by precisely forecasting the trends of the stock price indexes, small investors can protect themselves against external shocks and minimize their losses in a volatile environment.

Fundamental analysis and technical analysis are the two methods that have been developed for the prediction of stock indexes and for the formulation of investment choices. The fundamental analysis is a type of analysis of the intrinsic value of a stock based on various factors such as information available in a company's financial statements, the industrial factors and the economic trend. Technical analysis is based on the assumption that future movement follows some trends and these trends can be captured by using charts or some other tools to identify these patterns that are evaluated as the basis of an investment strategy. There is agreement among experts and analysts that the technical indicators used in technical analysis play a crucial role in forecasting the dynamic behavior of the stock market indexes. They are also critical in predicting the next day's trend whether the stock index is going up or down. By means of several simple rules, the turning points of these trends can be interpreted as a buy or sell decision for traders and investors in the 
market. Until now, technical indicators were mostly used by technical analysts and traders for profit-making. However, there are very few academic studies that used these technical indicators for forecasting the direction of stock price indexes (Liao \& Wang, 2010; Niaki \& Hoseinzade, 2013; Sheta et al., 2015; Dash \& Dash, 2016; Inthachot et al., 2016; Rajashree \& Pradipta, 2016)). With the increase of these studies, small investors and those who are concerned with financial markets can have a broader idea about technical analysis. Thus, they can take precautions against volatility and risks in stock market indexes.

In the past few decades, a great numbers of studies predicted stock market indexes. However, the nonlinear and nonstationary characteristics of the stock markets make it challenging and almost impossible to forecast stock markets in a reliable manner. Traditional statistical methods for forecasting financial time series are not very suitable to track the complex and nonstationary nature of the stock markets. In this regard, many researchers, traders and analysts have used various computational intelligence techniques to analyze the stock markets and to make trading decisions with higher prediction results. Such intelligence techniques include artificial neural network (ANN), rough set (RS), genetic algorithm (GA), fuzzy logic and others. Although all of these methods have unique advantages, they also have serious limitations that should be considered when conducting research and making analysis.

The integration of different intelligence techniques to overcome these limitations and to achieve synergic effects has contributed to a large number of new intelligent system designs through hybridization. In recent years, different kinds of intelligent system architectures have been developed and a growing number of studies have been conducted by using a variety of artificial intelligence techniques including ANN and GA in the stock index prediction. These studies generally try to estimate and compare the forecast accuracy of the artificial intelligence techniques with other traditional econometrical and statistical models. The results in general confirm that the intelligence techniques provide more accurate results as compared to traditional ones.

Although the number of studies conducted in emerging markets is a lot fewer than the developed ones, since the beginning of 2000s, a significant increase has been observed in the number of studies made on the emerging market indexes (Al-hnaity \& Abbod, 2016; Banik et al., 2014; Chang et al., 2012; Andre \& Tulio, 2007; Chen et al., 2003; Chatterjee, 2000; Lee \& Brahmasrene, 2018; Camba, 2020; Kakinuma, 2020; Sayed, 2014; Nguyen \& Nguyen, 2019). Furthermore, some researchers hybridized several intelligence techniques to predict the direction of daily price changes of stock markets. These studies also aim to confirm whether the hybridized techniques outperform each intelligence technique alone or not (Hassan et al., 2007;
Hawley et al., 1990; Holland, 1975; Hsieh, 1993; Inthachot et al., 2016; Kara et al., 2011; Kiani \& Kastens, 2008).

The primary aim of this paper is to predict the Borsa Istanbul (BIST) 30 index movements to determine the buy and sell decisions using the most popular and recently used computational intelligence methods of artificial neural network (ANN) and genetic algorithm (GA). Borsa Istanbul is the only stock exchange in Turkey, established in 1986 under the name of Istanbul Stock Exchange and renamed Borsa Istanbul in 2013. We prefer to conduct a research on the Borsa Istanbul (BIST) 30 index rather than Borsa Istanbul (BIST) 50 index or Borsa Istanbul (BIST) 100 index. This is because BIST 30 index has been formed by measuring the joint performance of the shares of the 30 companies traded in Borsa Istanbul that have the highest trading volumes and the highest market values. In order to determine the shares to be included in the BIST 30 index, the market values and the daily average transaction volumes of the shares in the actual circulation are ranked from large to small. In both rankings, the top rankers are taken into this index.

In this study, we combined the methods of artificial neural network (ANN) and genetic algorithm (GA) and obtained a hybrid intelligence method. We implemented this hybrid intelligence technique to the most widely used nine technical indicators that we selected used in technical analysis. By applying this hybrid model to each of these indicators, we forecasted the movements of the Borsa Istanbul (BIST) 30 index. By applying several simple rules to the movements of these predicted indicators, we tried to determine the most correct buy and sell decisions. The major contribution of this paper to the literature is to apply a hybrid model to such a large number of indicators and to show and to verify the predictability of a stock price index direction using ANN and GA. In the finance literature, there are not many studies on the prediction of stock values particularly to determine the buy and sell decisions. Although researchers have until recently conducted studies on the prediction of stock exchange values, there are very few studies on the stock index movements to determine the most correct buy and sell decisions using the methods of Artificial Neural Networks (ANN) and Genetic Algorithm (GA).

The paper is structured as follows. Section 2 explains the methodology of the research including the research data, technical indicators used in the study, and the prediction models. Section 3 presents and discusses the empirical results. Section 4 contains the concluding remarks.

\section{Methodology}

\subsection{Research Data}

We obtained the data from the Borsa Istanbul (BIST) 30 Index for a time period of more than 15 years (14.03.2001- 
13.06.2016), which includes 3,834 days in total. The data is comprised of the opening price, high and low price, closing price and the volume traded. Moving average convergence divergence (MACD), relative strength index (RSI), commodity channel index (CCI), momentum, directional movement index (DMI), stochastic oscillator, on-balance volume $(\mathrm{OBV})$, average directional movement index (ADX), and simple moving averages (3-day moving average, 5-day moving average, 10-day moving average, 14-day moving average, 20-day moving average, 22-day moving average, 50-day moving average, 100-day moving average, 200-day moving average) are the main technical indicators that we used in this research as they are the most commonly used ones in a financial analysis.

The buy or sell order signals separately occurred with the help of all these oscillators or indicators for each variable. In this research, we used 60 variables in total that have been derived from these nine main technical indicators mentioned above by computing them one by one. 14 of these 60 variables were presented in Table 1 according to the success order of the variables used.

\subsection{Technical Indicators}

Technical indicators are statistics used to measure current conditions and to predict financial or economic trends. They are widely used in technical analysis to forecast changes in stock trends or price patterns in any traded asset. There are more than 100 technical indicators used by financial analysts and experts. Using a single indicator may not generate a trading signal and determine an investment strategy for the analysts to predict future price movements correctly. Therefore, determining which indicator to use in which instrument will be a very important choice for the investor. Based on this approach, we wanted to benefit from the advantages of all these widely used technical indicators instead of using a single indicator, and we built and tested the hybrid model. The descriptions and calculations of the main technical indicators used in this study are as follows.

\subsection{Prediction Models}

The ANN model and the GA model have been employed in this research to forecast the buy and sell decisions in the BIST 30 index. Until recently, researchers have obtained successful results by using each model separately or by combining them in the field of finance like in other fields. Although they have been used in several areas including credit and bond valuation, security and asset portfolio management, determining optimal capital structure and forecasting, they have been very rarely used for the purpose of forecasting the buy and sell decisions in indexes. GA as well as ANN have been used to forecast stock indexes in the last few decades. Recently, some researchers choose to combine different artificial intelligence techniques including GA and ANN to obtain better results with higher successful rates (Faria et al., 2009; Kumar, 2009; Kashei \& Bijari, 2010; Maia \& Carvalho, 2011; Niaki \& Hoseinzade, 2013; Sheta et al., 2015; Oztekin et al., 2016). In this context, there are not many studies conducted on the prediction of Borsa Istanbul (BIST) Stock Exchange (Kara et al., 2011; Tektas \& Karatas, 2004; Kutlu \& Badur, 2009; Caliskan \& Deniz, 2015). Based on these successful results in applied literature, we used the following models to predict buying or selling decisions in the BIST 30 stock index. (a) ANN, (b) GA and (c) hybrid model of ANN and GA.

\subsubsection{Artificial Neural Networks (ANNs)}

ANNs are one of the recently developed methods that have been frequently used for the purpose of forecasting in different fields. Although neural network simulations are known to be a recent development, they were actually produced before the advent of computer in 1943 by McCulloch and Pitts (1943). Forecasting the future successfully is very critical for many disciplines and is also one of the fields of research in which ANN applications received a significant interest is finance.

Their data-driven and nonlinear characteristics of ANNs make them a promising alternative for forecasting. Although linear methods are easy to develop and understand, they also have many limitations in analyzing nonlinear relationships in data. ANNs have two major advantages in forecasting over linear methods: ANNs can even solve the most complex underlying relationships in real world problems. A neural network can learn and does not have to be reprogrammed (Mehrara et al., 2010).

An ANN can be defined as a layered network, which consists of one or more artificial neuron(s). A basic ANN structure consists of an input layer, hidden layer(s) and an output layer. The number of network layers depends on the complexity of the problem being modeled. Basically, the artificial neuron receives signals or inputs from other neurons or from the environment, processes a generally nonlinear operation by transmitting the signal to other connected neurons and produces output signals as the final result (Niaki \& Hoseinzade, 2013).

An ANN has basically three major components network topology, spreading activation method, and training mechanism. The network topology is the architecture of the ANN. The number of network layers, the number of nodes in each layer and the types of connections with each other are all major subjects of network topology (O'Connor \& Madden, 2006)

Feedforward networks and feedback networks are the two major types of neural networks that constitute 
the network topology or the architecture of the ANN. In feedforward network, the information flow is one directional from the input layer to the hidden layer(s), and then to the output layer. There is no information flow from the output layer to the hidden layer(s). In feedback networks, signals can travel in both directions by introducing loops in the network. Feedback networks, which are also referred to as recurrent networks, are powerful, interactive and dynamic types of neural networks (Rumelhart \& McClelland, 1986).

In the analysis of time series with non-linear structure, the most commonly preferred type is three-layered feedforward backpropagation neural network (Samanta \& Bordoloi, 2005). Studies indicate that multilayer neural networks have superior nonlinear analysis capabilities and are capable of learning more complex patterns by backpropagation learning. It is a classical learning algorithm widely used in layered feedforward ANNs. The neurons are organized in layers, which send their signals forward and the errors are propagated backwards. The network is fed with inputs and the desired outputs. Next, the error between the actual and the desired output is calculated with the purpose of reducing this error by adjusting the weights until ANN learns the training data (Shen \& Loh, 2004).

Training process may be supervised or unsupervised. In supervised learning, inputs and outputs are provided and the network processes the inputs to obtain the resulting output and it compares the obtained outputs with the desired ones. The signals are then propagated back through the system to teach it to approximate to provide the desired result. In unsupervised learning, the network is provided with inputs but not with the desired outputs. The system itself decides to choose which features to group within the input data. This is called self-organization or adoption (Sugumaran, 2008).

\subsubsection{Genetic Algoritnm (GA)}

A more recent method used in conducting financial analysis is the genetic algorithm (GA). It is particularly promising in modeling financial problems that do not fit into traditional linear forecasting. It has gained significant interest among the researchers especially for the purpose of improving the performance of artificial intelligence techniques. For instance, GA is used to select the neural network topology, to determine the optimal number of hidden layers and to process elements to improve the performance of ANN. Recent studies indicate that GA can also be used to improve the learning algorithm and to eliminate the irrelevant patterns of ANN (Tsai \& Wang, 2009).

GA principles were first proposed by Holland (1975) and developed by Goldberg (1989) and Koza (1994). The main aim of the researchers in using GA is to solve optimization problems such as determining the optimal weights for the related hybrid model. GA has significant differences from other conventional methods, which makes GA more efficient and effective in searching for the optimal solution. First of all, encoding and decoding discrete points is used in computing the strings in GA rather than using the original parameter values. Therefore, GAs compute logic operations better than their counterparts due to the adaptation of binary strings. Additionally, the prior information is not important for GA since there is no need for such information because the primary population is randomly generated (Wei, 2013).

To summarize, GA generates and evaluates an initial population, and reports the best of last population to find an optimal solution (Zhang, 2003). While the ability of GA to find a close optimal solution is important, it may not always guarantee to find one. However, it is a big advantage of GA that it generally forecasts accurately by obtaining acceptable alternatives in a relatively short period of time.

\subsubsection{The Hybridized Model with Genetic Algorithm and Neural Network Model (GAAN)}

Although ANNs have many advantages, they also have several limitations that should be considered. First of all, determining the optimal combination of the network parameters is difficult. Secondly, choosing the relevant features of an ANN is not an easy task. Finally, great volume of data is needed to obtain an accurate result. There are many studies, which used GA-based hybrid models to minimize the drawbacks of back propagation neural network. The results of many studies conclude that GA can enhance the accuracy of the ANN models and can decrease the time needed for experimentation. In this study, the GA algorithm is used to optimize the functioning of our model and to minimize the biases of the ANN model.

The main purpose in this study is to apply the hybridized model involving feedforward backpropagation neural network (BPN) and genetic algorithm (GA) to forecast the buying or selling decisions based on the BIST-30 index daily data. GA was hybridized with multilayered-feedforward backpropagation neural network to optimize the ANN's parameters and to improve the accuracy of the proposed model. In other words, by means of GA, we can determine the train data size that shows how many days should go back (500, 600, 700, 800, 900, 1000, etc.). Additionally, how many data should be given as input in every iteration, the ANN architecture, and which variables should be given as input data are other subjects that we can determine accurately thanks to GA. Since the number of subsets is huge, it would take too much calculation time to run them. GA as an algorithm, can overcome this difficulty and consequently, these computations are preferred to obtain the best subsets of input variables. 


\section{Results and Discussion}

We obtained 94 ANN structures that succeeded in a very good accuracy level of $75 \%$ and even higher percentages by using GA. When interpreted from a statistical point of view, GA adopted the buy or sell-simple moving average variable and momentum-buy or sell variable at the rate of $97 \%$ in the selection of the input variables. We conducted training data with the data available until 18.02.2015. Then we implemented verification with the data of the following 81 days from that date on. The statistical data related to these experimentations are seen in Table 1.

While the number of recurrences (the number of selections made by the GA) of the buysellSMA5 as the best input is 92 with a ratio of $97,87 \%$, interestingly, macd. indicator has been the least recurred input with the number of recurrences of only 1 with a ratio of $1,06 \%$.

The data size and the ratios selected by the GA among the best 94 ANNs obtained are shown in Table 2. Accordingly, the parameter, which has been selected as train data size among the best 94 ANNs obtained, has been 500 days with a ratio of $91,49 \%$.

Table 3 indicates what our success rate can be when we forecast by going how many days back in the ANN. The table reveals that out of 94 ANNs, 91 of them were forecasted by going 22 days back. Thus, 22-day prediction has been preferred by the great majority of the ANNs.

Figure 1 shows the structure of the best model for the feedforward backpropagation genetic algorithm-neural network architecture with a successful forecast rate of $75 \%$. We used three input variables, namely, momentum buy/sell signal, 5-day simple moving average (SMA 5-day), and the OBV. Each input variable has 15 neurons and the input layer consists of 45 neurons in total. As seen from Figure 1, there are two hidden layers, the first one with 15 neurons and the second one with 10 neurons. The output layer is composed of buy/sell signal. This structure, which shows the best ANN model selected by the GA, has a successful forecast rate of $75 \%$, which is one of the highest rates obtained by a hybrid model of GA and ANN to forecast a stock price index in the applied literature until now. For instance, Inthachot et al. (2016) attained an average prediction accuracy rate of $63,60 \%$, which is one of the highest average prediction accuracy rates in the literature until now.

A hybrid intelligence model of ANN and GA was developed for determining buying and selling decisions based on the BIST-30 stock price index data over the period from 28.12.2015 to 13.06.2016. We forecasted nine main indicators by using these data that are shown in Figures 2 to 9 . These predicted technical indicators can be compared with the realized index values which is presented in Figure 2.

Moving average convergence divergence (MACD), which was presented in Figure 2, is a trend following and
Table 1: The number and the ratio of the best 94 ANNs which were selected by the GA

\begin{tabular}{|l|l|c|c|}
\hline & Name of the Input & $\begin{array}{c}\text { The Number of } \\
\text { Selections made } \\
\text { by the GA }\end{array}$ & $\begin{array}{c}\text { Ratio } \\
\text { (\%) }\end{array}$ \\
\hline 1 & buysellSMA5 & 92 & 97,87 \\
\hline 2 & momentum.buysell & 92 & 97,87 \\
\hline 3 & dmi.buysell3 & 90 & 95,74 \\
\hline 4 & macd.buysel & 87 & 92,55 \\
\hline 5 & price & 77 & 81,91 \\
\hline 6 & rsi.indicator & 71 & 75,53 \\
\hline 7 & cci.indicator & 70 & 74,47 \\
\hline 8 & dmi.buysell1 & 70 & 74,47 \\
\hline 9 & cci.buysell3 & 70 & 74,47 \\
\hline 10 & ma.SMA5 & 69 & 73,40 \\
\hline 11 & momentum. & 68 & 72,34 \\
\hline 12 & indicator2SMA & 67 & 71,28 \\
\hline 13 & obv.buysell1 & 66 & 70,21 \\
\hline 14 & obv.indicator & 61 & 64,89 \\
\hline
\end{tabular}

Table 2: The data size and the ratios selected by the GA among the best 94 ANNs obtained

\begin{tabular}{|l|c|c|c|}
\hline & DATA_SIZE & Number & Ratio(\%) \\
\hline 1 & 400 & 1 & 1,06 \\
\hline 2 & 300 & 3 & 3,19 \\
\hline 3 & 600 & 4 & 4,26 \\
\hline 4 & 500 & 86 & 91,49 \\
\hline
\end{tabular}

Table 3: The selection ratios of the input window sizes among the best 94 ANNs obtained

\begin{tabular}{|l|c|c|c|}
\hline & INPUT_WINDOW_SIZE & Number & Ratio (\%) \\
\hline 1 & 30 & 2 & 2,13 \\
\hline 2 & 22 & 91 & 96,81 \\
\hline 3 & 10 & 1 & 1,0 \\
\hline
\end{tabular}

momentum indicator that fluctuates above and below zero. The upper part of the figure indicates the realized values of BIST 30 index between the mentioned dates above. Just below the realized values, we see the blue line and the red line. They are two different types of MACD lines. The red one is the MACD trigger, which represents the faster exponential moving average and the blue one is MACD indicator, which represents the slower exponential moving average. 


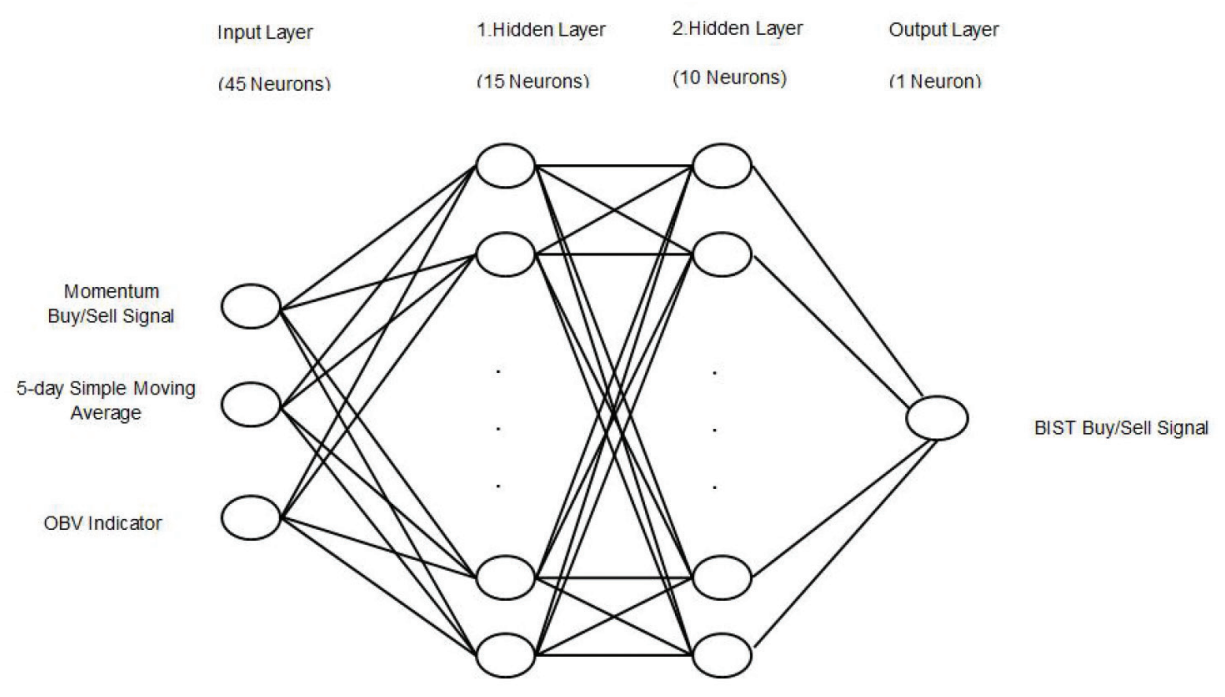

Figure 1: The best model for the feedforward backpropagation genetic algorithm - neural network architecture

Many analysts interpret that potential buy signals occur when the MACD line moves above zero, and potential sell signals occur when it falls below zero. Additionally, it can be said that, if MACD indicator line is above the MACD trigger line, then positive trend is prevalent. On the contrary, if MACD indicator line is below the MACD trigger line, then negative trend is prevalent. For buying decisions, price bottoms and indicator bottoms are compared, whereas for selling decisions price tops and indicator tops are compared. According to the figure, between the dates of 21.04.2016 and 13.05.2016, as MACD trigger line is above MACD indicator line, a buy signal occurs. On the other hand, the dates between 13.05.2016 and 13.06.2016 indicate the area where MACD indicator line is above MACD trigger line and consequently, a sell signal occurs. In general, traders agree that, if prices rise too high suddenly, there is a relatively small probability that the trend can be sustainable at those elevated levels. Similarly, if prices fall too low, too quickly, there is a small chance that prices can keep at those historically inexpensive levels. Finally, we can see the histogram format of the MACD trigger and MACD indicator line in the lower part of the figure.

Two types of commodity channel indexes (CCI) have been presented in Figure 3. For the interpretation of the CCI, +100 and -100 lines are important rather than the zero line. When the CCI line intersects the +100 line upward, it can be interpreted as buy signal. However, if it returns back to +100 , then it can be interpreted as sell signal. When the CCI intersects the -100 downward, it is evaluated as short sell signal and when it comes back to -100 then it is evaluated as buy signal. The higher the CCI signal, the stronger the uptrend. The lower the CCI signal, the stronger the downtrend. The crossovers of both lines may constitute buy and sell signals and we can realize them by drawing trend lines on CCI.

Results of the relative strength index (RSI) are shown in Figure 3 at the bottom. It is an index, which aims to determine the overbought and the oversold levels. The markets are evaluated as overbought above 70 and oversold below 30 . Figure 3 indicates that there are two strong sell signals between the dates of 10.03.2016 and 24.03.2016 as the RSI approaches to the level of 80 and then moves downward to the level of 70 twice. If the RSI reaches over 70 and makes a peak and then reverses from that point, then it can be termed as unsuccessful waves.

Three different momentum indicators are presented in Figure 4. When traders want to use momentum indicator, they usually take a long position in a stock or asset that is trending. However, they prefer to take short position if the stock is trending down. Instead of traditional trading strategy of buy low and sell high, momentum investing strategy is based on selling low and buying lower or buying high and selling higher. Therefore, instead of identifying the continuation or reversal pattern, traders using this indicator focus on the trend created by the most recent price break.

The directional movement indicator (DMI), which is used to confirm price action, can also be seen from Figure 4. In the upper part of the figure, the indicators of DIOSC and DIOSC +sma can be seen. While the tops of these indicators are interpreted as sell signals, the bottoms of them are interpreted as buy signals. Divergence with the realized values is also useful in making correct predictions. 

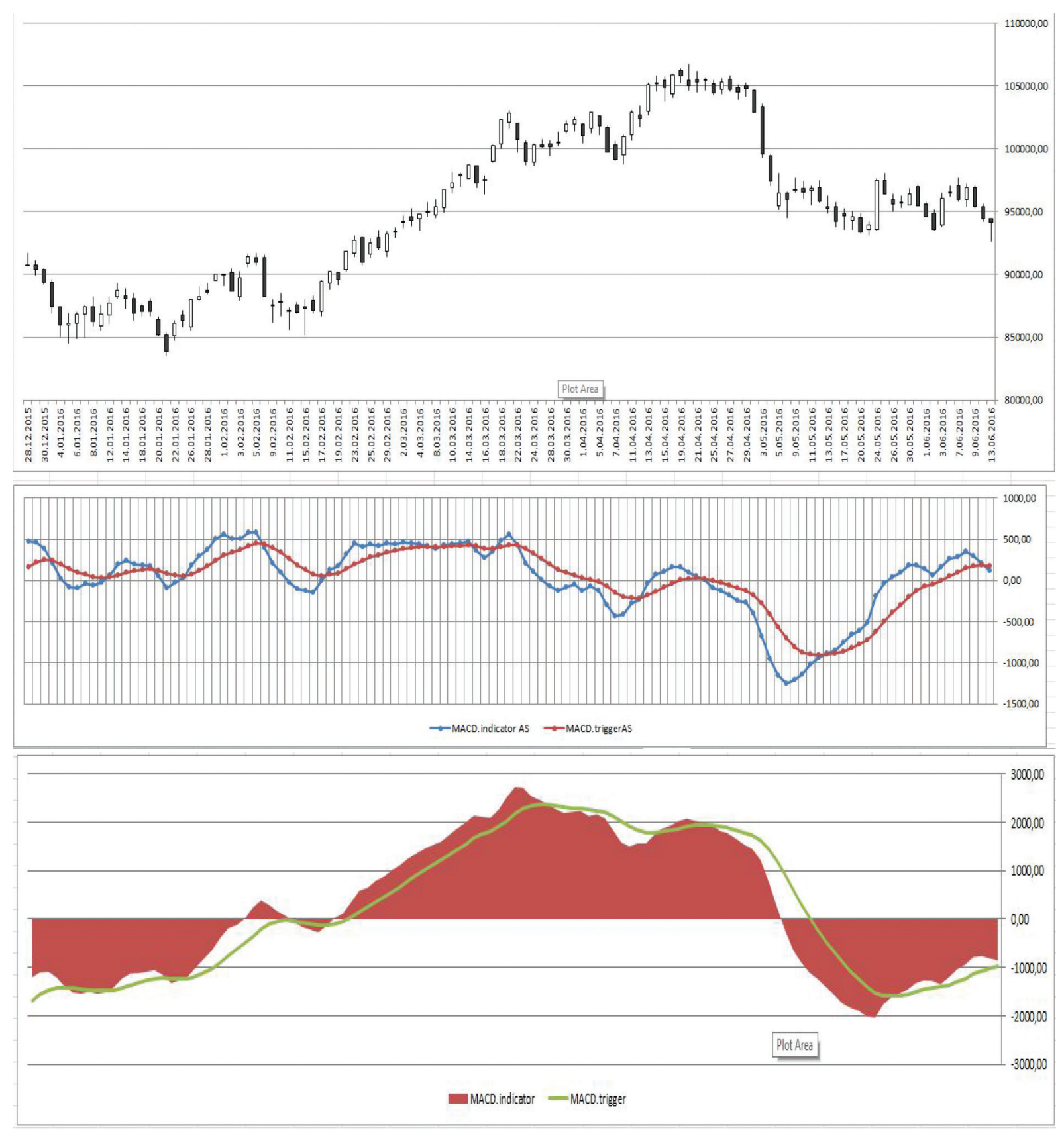

Figure 2: The interpretation of MACD

In the lower part of Figure 5, we see the +DI and -DI. In general, + DI moves in synchronization with price which means that it rises when price rises and falls when price falls. However, -DI behaves in the opposite manner and moves counter directional to the price. In Figure 5, when + DI breaks through the -DI in an upward direction it gives a buy signal. On the contrary, when -DI breaks through the +DI in an upward direction it gives a sell signal.
The stochastic oscillator is an important indicator used very frequently in identifying overbought and oversold levels. Within the range from 0 to 100 , the most traditional settings for the oscillator, 20 is typically the oversold threshold and 80 is the overbought threshold. It is generally interpreted that a buy signal is formed when the $\% \mathrm{~K}$ breaks through the $\% \mathrm{D}$ in an upward direction. In contrast, a sell signal is formed when the $\% \mathrm{~K}$ falls through the $\% \mathrm{D}$ in a downward direction. 

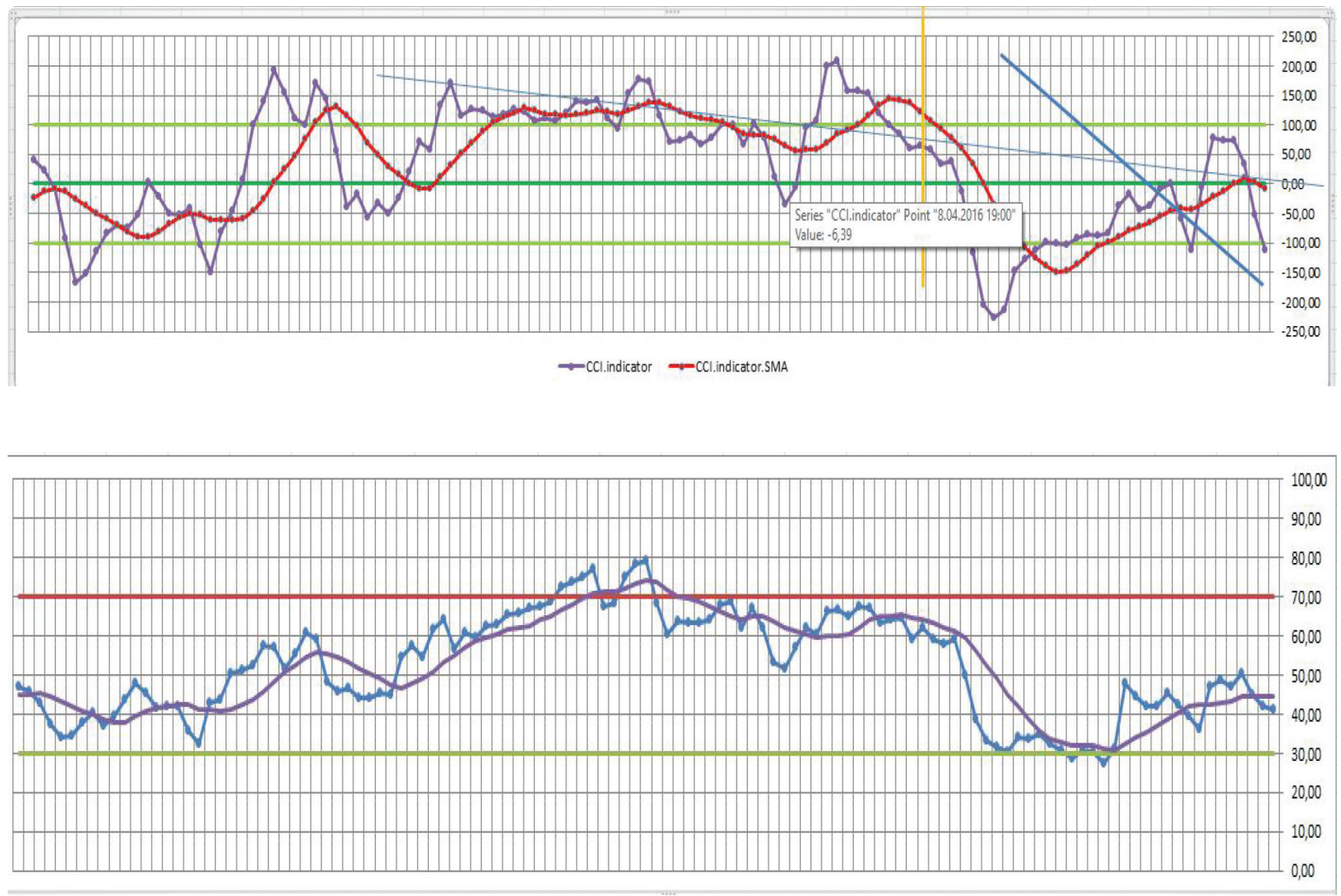

Figure 3: The interpretation of $\mathrm{CCl}$ and $\mathrm{RSI}$

Divergence may also be helpful in identifying reversals. The shape of a stochastic bottom and top are also good indicators as to whether the trend will continue or not. In Figure 5, in the determined period of time, we recognize that the movement over 80 occurs three times, which indicates an overbought level. On the contrary, the movement below 20 occurs only once, which indicates an oversold level. In those areas, many buy and sell signals are obtained based on the movements and the directions of $\% \mathrm{~K}$ and $\% \mathrm{D}$ with each other.

The average directional index (ADX) is a nondirectional index, which quantifies the trend strength by means of both uptrends and downtrends. As can be seen from Figure 6, when +DI is above the -DI, prices generally move up and the ADX measures the strength of the uptrend. In contrast, when the -DI is above the +DI, prices generally move downward. In this regard, Figure 6 is an example of an uptrend reversing to a downtrend. We can notice how ADX rises during the uptrend when the $+\mathrm{DI}$ is above the -DI. When price reverses, the -DI crosses above the $+\mathrm{DI}$, and ADX rises again, this time to measure the strength of the downtrend. It is generally agreed by the traders that an ADX value of 25 and less indicates an absent or weak trend, whereas when it rises above 25 , price tends to trend.

The simple moving average (SMA) is one of the simplest and the most widely used indicator in technical analysis. It is the simple average of a security over a defined number of time periods. For instance, a 5-day MA will be calculated by adding the last 5 days together and then dividing by 5 . It is a trend-following or lagging indicator because it is based on past prices. The most common applications of them are to identify the trend direction and to determine support and resistance levels. In Figure 6, the price, 5-day SMA and 22-day SMA have been presented. It is striking that while the 5-day SMA follows a very parallel trend to the realized prices, 22-day SMA follows somewhat a different trend than the actual prices. 

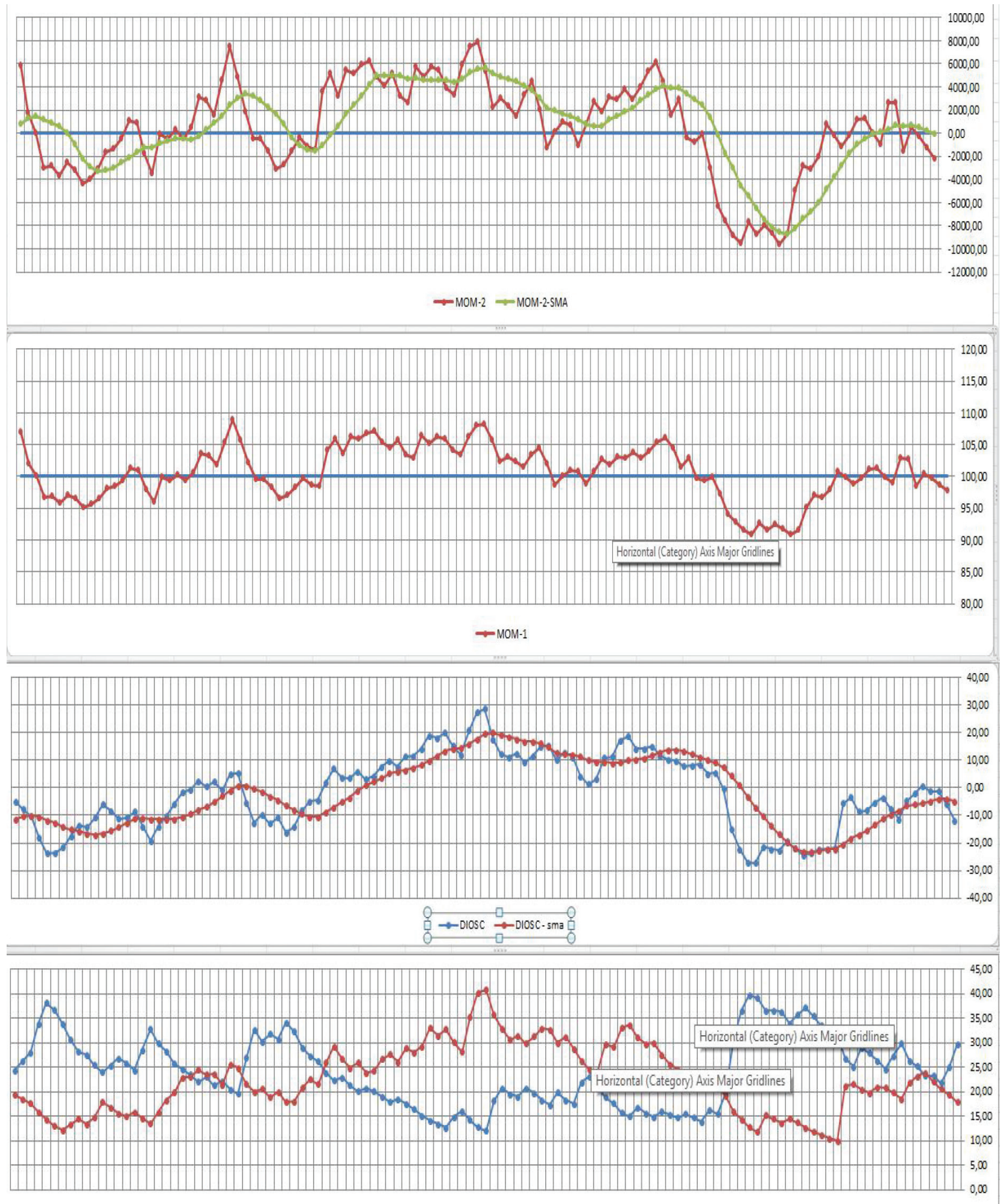

$\rightarrow-D 1 \rightarrow+D \mid$

Figure 4: The interpretation of momentum and DMI 

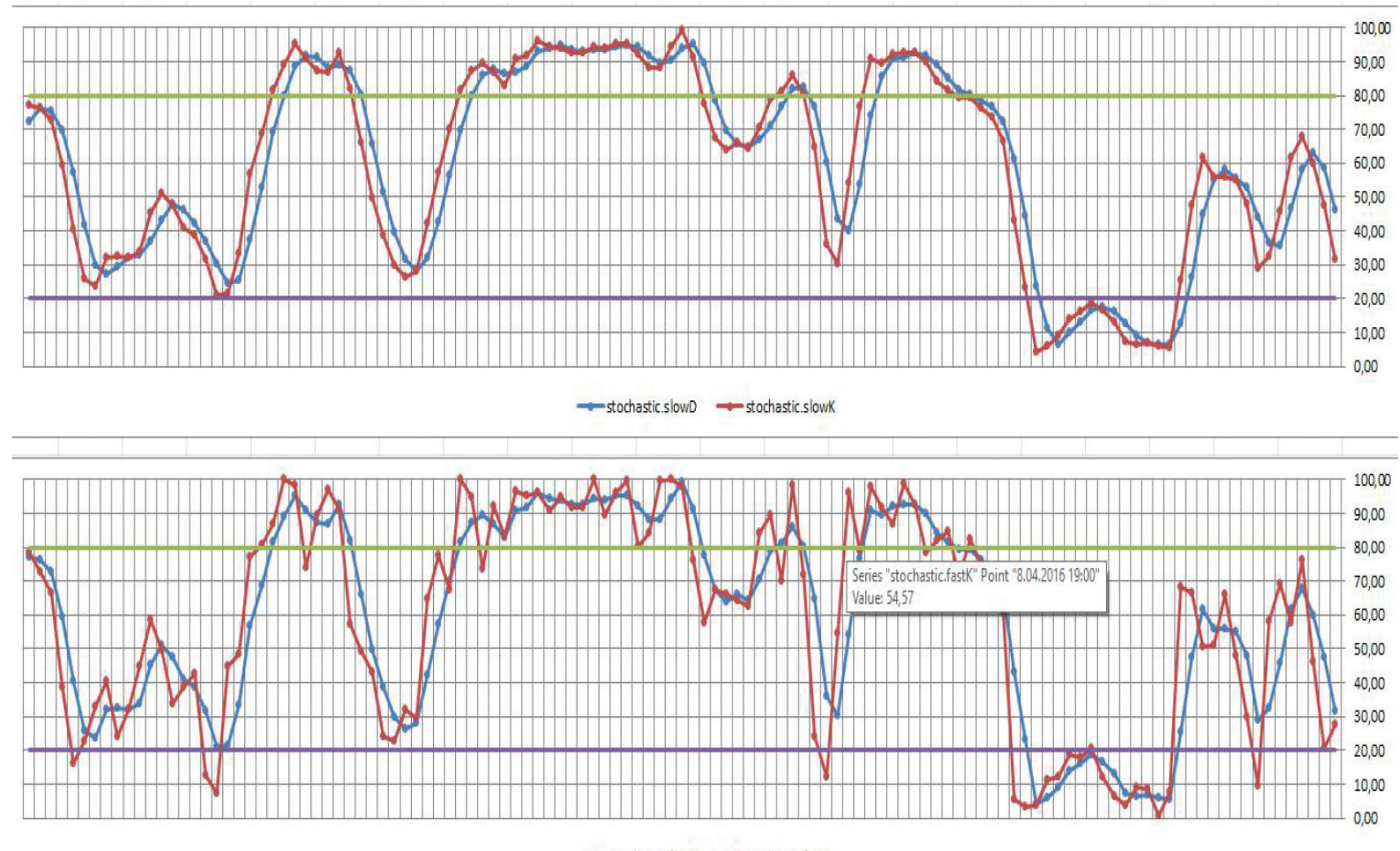

Figure 5: The interpretation of stochastic oscillator

\section{Conclusions}

Forecasting a stock market has been a difficult task due to its nonlinear, dynamic and complex nature, but when it is achieved, it can provide huge profits to those who could do it. Thus, proper forecast of the market has utmost importance for investors, traders, fund managers, researchers, and many others who are interested in investing. In the last few decades, machine-learning techniques have been effective in stock forecasting. In this context, use of algorithms in trading increased after computerized trading systems were introduced in many of the financial markets since 1970s. By 2014, more than 70 percent of all trades were executed by computers at the New York Stock Exchange (NYSE).

Usually, in a stock market, traders use either fundamental analysis or technical analysis to successfully identify the point where they will play the stock markets, while deciding on the direction of investment with basic analysis knowledge. Today, it is possible to produce successful results with technical analysis data by using different technical indicators. In this research, we used the most important technical indicators and compared them with the realized index values. Rather than using a single technical indicator, we obtained nine figures from these nine different indicators. By using our main indicators that we selected, we designed a hybrid intelligence model of ANN and GA for predicting optimal buy and sell time and for making the best decisions. We tested the hybrid models and selected the best one by genetic algorithm. The ANN prediction model and the GA prediction model were combined to improve the prediction performances of ANN and GA alone to provide the most correct decisions whether to buy, sell or hold a stock.

We obtained the data used in this study from the Borsa Istanbul (BIST) 30 Index for a time period of more than 15 years (14.03.2001 - 13.06.2016), which includes 3,834 days in total. The data is comprised of the opening price, high and low price, closing price and the volume traded. Rather than concentrating on a single or few indicators, this study tried to include most of the widely used technical indicators to achieve a superior forecast precision. In this research, we used 60 variables in total that have been derived from the nine main technical indicators we used by computing one by one. The experimental result indicates that our best proposed hybrid model has a successful forecast rate of $75 \%$, which is higher than the single ANN or GA forecasting models. This prediction rate is also one of the highest forecast results 

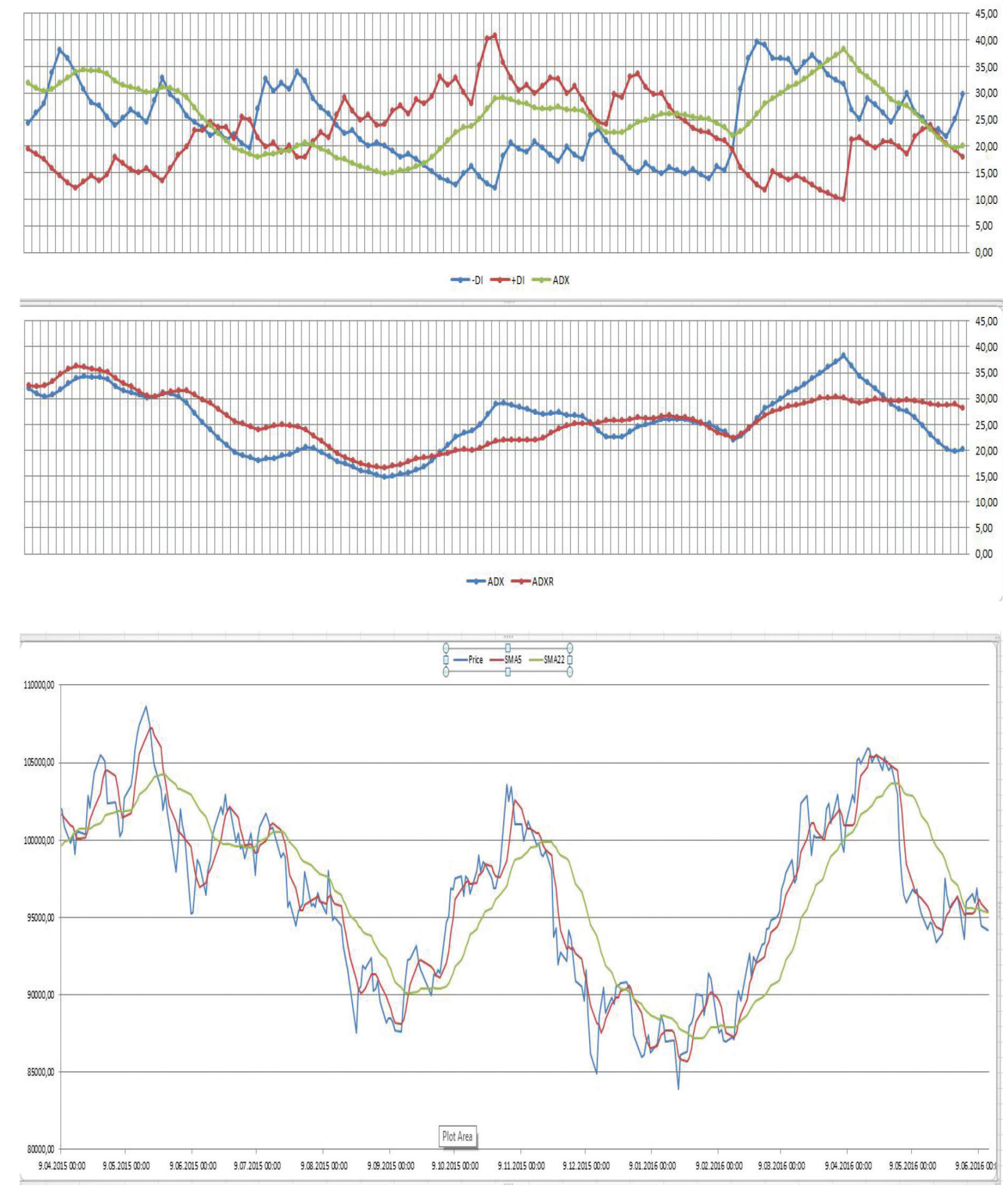

Figure 6: The Interpretation of ADX and SMA 
in various papers in our literature survey that used hybrid models of GA and ANN for stock price index prediction. As a suggestion for future work, other forecasting models using different artificial intelligence techniques as part of hybrid approaches can also be used to get better forecast results. The addition of other technical indicators may also improve the quality of the models in future research. The stock index direction can also be predicted by using deep learning techniques with the same or similar indicators.

\section{References}

1-hnaity, B., \& Abbod, M. (2016). Predicting Financial Time Series Data Using Hybrid Model. In: Y. Bi et al. (Eds.), Intelligent Systems and Applications (pp. 19-41). Cham, Switzerland: Springer International Publishing.

Andre, C., \& Tulio, R. (2007). Do artificial neural networks provide better forecasts? Evidence from Latin American stock indexes. Latin American Business Review, 8(3), 92-110.

Banik, S., Khan, A., \& Anwer, M. (2014). Hybrid machine learning technique for forecasting Dhaka Stock Market timing decisions, Computational Intelligence and Neuroscience, 2014, 1-6. https://doi.org/10.1155/2014/318524

Caliskan, M. M. T., \& Deniz, D. (2015). Yapay Sinir Aglarıyla Hisse Senedi Fiyatlari ve Yonlerinin Tahmini. Forecasting the directions and prices of stocks by using artificial neural networks. Journal of the Faculty of the Economics and Adminstrative Sciences of Eskisehir Osmangazi University, 10(3), 177-194.

Camba, A. C. (2020). Capturing the Short-run and Long-run Causal Behavior of Philippine Stock Market Volatility under Vector Error Correction Environment. Journal of Asian Finance, Economics and Business, 7(8), 41-49. doi:10.13106/jafeb.2020. vol7.no8.041

Chang, P., Wang, D., \& Zhou, C. (2012). A novel model by evolving partially connected neural network for stock price trend forecasting. Expert Systems with Applications, 39, 611-620.

Chatterjee, A. (2000). Artificial nural network and the financial markets: a survey. Managerial Finance, 26(12), 32-45.

Chen, A., Leung, M., \& Daouk, H. (2003). Applications of neural networks to an emerging financial market: Forecasting and trading the Taiwan Stock Index. Computers and Operations Research, 30(6), 901-923.

Dash, R., \& Dash, K. P. (2016). A hybrid stock trading framework integrating technical analysis with machine learning techniques. The Journal of Finance and Data Science, 4(1), 42-57.

Faria, E. L., Albuqurque, M. P., Gonzales, J. L., Cavalcante, J. T. P., \& Albuqurque, M. P. (2009). Predicting the Brazilian stock market through neural networks and adaptive exponential smoothing methods. Expert System with Applications, 36(10), 12506-12509.
Goldberg, D. E. (1989). Genetic Algorithms in Search, Optimization, and Machine Learning. Boston, MA: Addison Wesley Longman Publishing.

Hassan, M. R., Nath, B., \& Kirley, M. (2007). A fusion model of HMM, ANN and GA for stock market forecasting. Expert Systems with Applications, 33, 171-180.

Hawley, D., Johnson, J., \& Raina, D. (1990). Artificial neural systems: a new tool for financial decision-making. Financial Analysts Journal, 46(6), 63-72.

Holland, J. (1975). Adaptation in Natural and Artificial Systems. Ann Arbor, MI: University of Michigan Press.

Hsieh, C. T. (1993). Some potential applications of artificial neural systems in financial management. Journal of Systems Management, 44(4), 12-15.

Inthachot, M., Boonjing, V., \& Intakosum, S. (2016). Artificial neural network and genetic algorithm hybrid intelligence for predicting Thai Stock Price Index. Computational Intelligence and Neuroscience, 2016, 1-8. https://doi. org/10.1155/2016/3045254

Kakinuma, Y. (2020). Return Premium of Financial Distress and Negative Book Value: Emerging Market Case. Journal of Asian Finance, Economics and Business, 7(8), 25-31. doi:10.13106/ jafeb.2020.vol7.no8.025

Kara, Y., Boyacioglu, M. A., \& Baykan, O. K. (2011). Prediction of stock price index movement using artificial neural networks and support vector machines: the sample of the Istanbul Stock Exchange. Expert Systems with Applications, 38, 5311-5319.

Kashei, M., \& Bijari, M. (2010). An artificial neural network (p, d, q) model for timeseries forecasting. Expert Systems with Applications, 37(1), 479-489.

Kiani, K., \& Kastens, T. (2008). Testing forecast accuracy of foreign exchange rates: predictions from feed forward and various recurrent neural network architectures. Computational Economics, 32(4), 383-406.

Koza, J. R. (1994). Genetic Programming II: Automatic Discovery of Reusable Programs. Cambridge, MA: MIT Press.

Kumar, M. (2009). Nonlinear Prediction of the Standard and Poor's 500 and the Hang Seng Index Under a Dynamic Increasing Sample. Asian Academy of Management Journal of Accounting and Finance, 5(2), 101-118.

Kutlu, B., \& Badur, B. (2009). Yapay Sinir Aglari ile Borsa Endeksi Tahmini. Stock Market Index Prediction with Artificial Neural Networks. Journal of Yonetim, 20(63), 25-40.

Lee, J. W., \& Brahmasrene, T. (2018). An Exploration of Dynamical Relationships between Macroeconomic Variables and Stock Prices in Korea. Journal of Asian Finance, Economics and Business, 5(3), 7-17. doi:10.13106/jafeb.2018.vol5.no3.7

Liao, Z., \& Wang, J. (2010). Forecasting model of global stock index by stochastic time effective neural network, Expert Systems with Applications, 37(1), 834-841. 
Maia, A., \& de Carvalho, F. (2011). Holt's exponential smoothing and neural network models for forecasting interval-valued time series. International Journal of Forecasting, 27, 740-759.

McCulloch, W., \& Pitts, W. (1943). A logical calculus of the ideas immanent in nervous activity, The Bulletin of Mathematical Biophysics, 5(4), 115-133.

Mehrara, M. M., Ahrari, A., \& Ghafari, A. (2010). Using technical analysis with neural network for prediction stock price index in Tehran Stock Exchange, Middle Eastern Finance and Economics, 6(6), 50-61.

Nguyen, C. T., \& Nguyen, M. H. (2019). Modeling Stock Price Volatility: Empirical Evidence from the Ho Chi Minh City Stock Exchange in Vietnam. Journal of Asian Finance, Economics and Business, 6(3), 19-26. https://doi.org/10.13106/ jafeb.2019.vol6.no3.19

Niaki, S. T. A., \& Hoseinzade, S. (2013). Forecasting S\&P 500 index using artificial neural networks and design of experments, Journal of Industrial Engineering International, 9(1), 1-9.

O'Connor, N., \& Madden, M. (2006). A neural network approach to predicting stock exchange movements using external factors, Knowledge-Based Systems, 19, 371-378.

Rajashree, D., \& Pradipta, K. D. (2016). A hybrid stock trading framework integrating technical analysis with machine learning techniques. The Journal of Finance and Data Science, 4(1), 42-57.

Rumelhart, D., \& McClelland, J. (1986). Parallel Distributed Processing: Explorations in the Microstructure of Cognition. Cambridge, MA: MIT Press.

Samanta, G. P., \& Bordoloi, S. (2005). Predicting stock market: an application of artificial neural network technique through genetic algorithm. Finance India, 19(1), 173-188.
Sayed, S. A. (2014). Do Analyst Practices and Broker Resources Affect Target Price Accuracy? An Empirical Study on Sell Side Research in an Emerging Market. Journal of Asian Finance, Economics and Business, 1(3), 29-36. doi: 10.13106/ jafeb.2014.vol1.no3.29.

Sevim, C., Oztekin, A., Bali, O., Gumus, S., \& Guresen, E. (2014). Developing an early warning system to predict currency crises. European Journal of Operational Research, 237(1), 1095-1104.

Shen, L., \& Loh, T. T. (2004). Applying rough sets to market timing decisions. Decision Support Systems, 37(4), 583-597.

Sheta, A., Ahmet, S., \& Faris, H. (2015). A comparison between regression artificial neural networks and support vector machines for predicting stock market index, International Journal of Advanced Research in Artificial Intelligence, 4(7), 55-63.

Sugumaran, V. (2008). Intelligent Information Techologies: Concepts, Methodologies, Tools, and Applications. Hershey, PA: IGI Global.

Tektas, A., \& Karatas, A. (2004). Artificial Neural Networks and Their Applications in the Field of Finance: Prediction of Stock Prices. Ataturk University Journal of Economics and Administrative Sciences, 3(3-4), 337-349.

Tsai, C., \& Wang, S. P. (2009). Stock price forecasting by hybrid machine learning techniques. In: Proceedings of the International Multiconference of Engineers and Computer Scientists, IMECS 2009, Hong Kong.

Wei, L. (2013). A Hybrid Model Based on ANFIS and Adaptive Expectation Genetic Algorithm to Forecast TAIEX. Economic Modelling, 33, 893-899.

Zhang, P. (2003). Time series forecasting using a hybrid ARIMA and neural network model. Neurocomputing, 50, 159-175. 